\title{
Targeting BRAF mutations in non-small cell lung cancer
}

\author{
Connor Gerard O’Leary ${ }^{1,2}$, Vladamir Andelkovic ${ }^{1}$, Rahul Ladwa ${ }^{1}$, Nick Pavlakis ${ }^{3}$, Caicun Zhou ${ }^{4}$, \\ Fred Hirsch $^{5}$, Derek Richard ${ }^{2}$, Kenneth O'Byrne , $^{1,2}$
}

${ }^{1}$ Princess Alexandra Hospital, Brisbane, Australia; ${ }^{2}$ Translational Research Institute, Brisbane, Australia; ${ }^{3}$ Royal North Shore Hospital, Sydney, Australia; ${ }^{4}$ Shanghai Pulmonary Hospital, Shanghai, China; ${ }^{5}$ Mount Sinai Hospital, New York, NY, USA

Contributions: (I) Conception and design: CG O’Leary, K O’Byrne; (II) Administrative support: None; (III) Provision of study materials or patients: None; (IV) Collection and assembly of data: CG O'Leary; (V) Data analysis and interpretation: CG O’Leary, K O’Byrne; (VI) Manuscript writing: All authors; (VII) Final approval of manuscript: All authors.

Correspondence to: Dr. Connor Gerard O’Leary. Department of Medical Oncology, Princess Alexandra Hospital, 199 Ipswich Road, Woolloongabba, Brisbane, Queensland 4102, Australia. Email: connor.oleary@health.qld.gov.au.

\begin{abstract}
The management of non-small cell lung cancer (NSCLC) has changed significantly with the discovery of specific drug targets. These drugs have helped transform patient care and outcomes. BRAF mutated NSCLC is now recognised as a rare form of lung cancer. Data has begun to emerge supporting the use of BRAF/MEK inhibitors that target $\mathrm{BRAF}^{\mathrm{V} 600 \mathrm{E}}$ mutations in the mitogen-activated protein kinase (MAPK) pathway. Multiple phase 2 studies have been performed assessing the effectiveness of single agent BRAF inhibition and combination BRAF/MEK inhibition in pretreated and untreated patient populations. Consistently overall response rate (ORR) and progression free survival (PFS) are improved with the addition of a MEK inhibitor. A 2-cohort phase 2 study demonstrated an ORR of 33\% vs. $67 \%$ and PFS of 5.5 vs. 10.2 months in those treated with single agent dabrafenib $v$ s. dabrafenib and trametinib respectively. A similar ORR of $63 \%$ and PFS of 10.9 months was seen in a separate phase 2 study in patients treated with Dabrafenib and Trametinib in the first line setting. Immunotherapy is beginning to show promise as an active therapy in BRAF mutated NSCLC in both V600E and non-V600E subtypes; however, this requires further study and clarification. BRAF ${ }^{\mathrm{V} 600 \mathrm{E}}$ mutated NSCLC treated with chemotherapy have been widely reported to be associated with worse outcomes when compared to those without a mutation. With efficacy of combination BRAF/MEK established and early evidence of immune checkpoint inhibitor activity careful consideration should be given when choosing the most appropriate therapy in this select patient cohort.
\end{abstract}

Keywords: Non-small cell lung cancer (NSCLC); dabrafenib; trametinib; vemurafenib; BRAF ${ }^{\text {V600E }}$

Submitted Jan 14, 2019. Accepted for publication Oct 12, 2019.

doi: $10.21037 /$ tlcr.2019.10.22

View this article at: http://dx.doi.org/10.21037/tlcr.2019.10.22

\section{Introduction}

A dramatic evolution in lung cancer treatment has occurred over the last decade with the discovery of multiple specific druggable targets. The most notable include epidermal growth factor receptor (EGFR) exon 19 deletion and exon 21 L858r mutation, anaplastic lymphoma kinase (ALK) translocation and ROS-1 rearrangement. These developments have allowed for a more personalised approach to lung cancer treatment in a select patient cohort. Drugs that are active at these sites have translated into a significant improvement in patient survival and quality of life compared to conventional cytotoxic therapies (1-4). With the success of BRAF targeting drugs observed in other cancer types attention has now turned to the subgroup of lung cancer patients with BRAF mutations. Promising data is starting to emerge that supports the use of these drugs in this setting.

\section{Pathological and clinical characteristics}

The BRAF kinase is an essential step in intracellular 


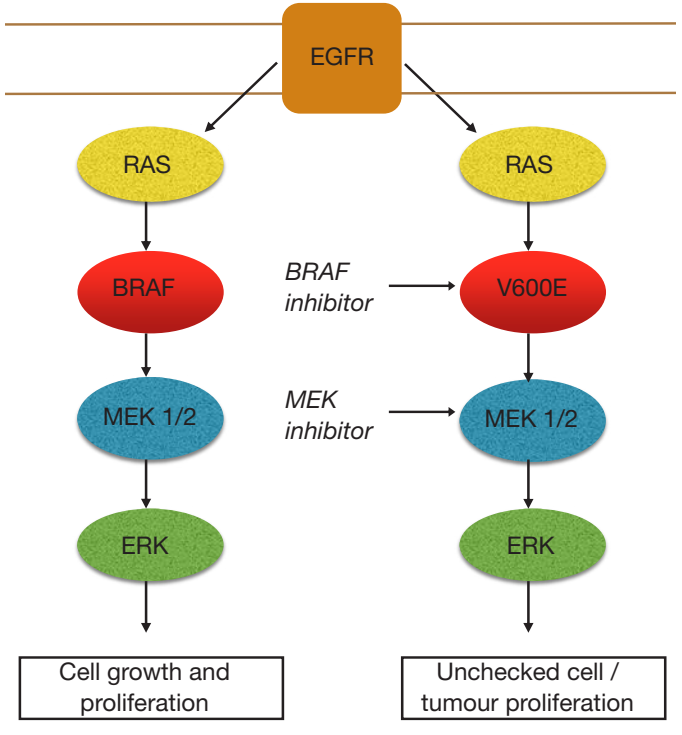

Figure 1 MAPK pathway-left: normal pathway, right: V600E mutation and target sites for BRAF and MEK inhibitors.

signalling which facilitates signal transmission from the cell surface to the nucleus after activation of the EGFR. BRAF is part of the mitogen-activated protein kinase (MAPK) pathway which plays a major role in controlling cell growth and regulation. In healthy tissue the BRAF kinase is deactivated through negative feedback once the signal has moved on to the next point in the cascade. BRAF mutations occurring in the MAPK pathway results in persistent activation of downstream cell signalling. BRAF mutations acting in this manner behave as oncogenic drivers which can lead to unchecked cell growth and proliferation $(5,6)$ (Figure 1). The most commonly described BRAF variant responsible for this process is a BRAF point mutation, V600E (7). This point in the cell pathway have emerged as a valuable therapeutic target for drug therapy with BRAF inhibitors. Resistance invariably develops with the development of bypass pathways such as redirection of cell signalling via MEK $1 / 2$ kinases. The addition of a MEK inhibitor in combination with a BRAF inhibition has resulted in improved clinical outcomes in some cancer types with BRAF mutations. This, however, does not ultimately prevent resistance developing in the vast majority of cases $(8,9)$. Acquired resistance post exposure to BRAF/MEK inhibition remains the most challenging aspect of treating patients with these drugs. Activation and cross talk between parallel pathways are regarded as a common mechanism that tumour cells use to overcome the blockade imposed by the
BRAF/MEK inhibitors. The PI3K/AKT/mTOR pathway is intrinsically linked to the MAPK pathway. In the setting of BRAF/MEK inhibition cell signalling is up regulated over time through this pathway allowing ongoing tumour progression through this alternate pathway. Preliminary study is underway attempting to develop effective drug combinations to target this pathway $(10,11)$.

BRAF mutations are rare in non-small cell lung cancer (NSCLC), occurring in $1-5 \%$ of cases. BRAF mutations are most commonly identified in lung adenocarcinoma. $\mathrm{BRAF}^{\mathrm{V} 600 \mathrm{E}}$ is consistently described as the most common variant identified, present in more than $50 \%$ of cases with a BRAF mutation (12-15). A number of large studies have been performed looking at the clinical and pathological features associated with these tumours. Some variability has emerged relating to the specific tumour associations identified from these studies (Table 1).

A study of 1,680 Chinese patients reported an association between BRAF and never smoking history $(\mathrm{P}=0.019)$ and a slight majority of female cases $(\mathrm{P}=0.682)$. They observed shorter progression free survival in the $\mathrm{BRAF}^{\mathrm{V} 600 \mathrm{E}}$ cohort treated with platinum-based chemotherapy however this did not reach statistical significance, 5.2 vs. 6.4 months $(\mathrm{P}=0.561)(15)$. A similar study performed in a North American Caucasian population included 883 patients with NSCLC. In this study, there was similar rates of $\mathrm{BRAF}^{\mathrm{V} 600 \mathrm{E}}$ and non $\mathrm{BRAF}^{\mathrm{V} 600 \mathrm{E}}$ mutations identified in males and females. Over two thirds of those with BRAF mutations were current or former smokers. Similar to their Chinese counterparts this study also identified a shorter progression free survival for patient harbouring a $\mathrm{BRAF}^{\mathrm{V} 600 \mathrm{E}}$ mutation when treated with platinum-based chemotherapy, $4.1 \mathrm{vs}$. 8.9 months $(\mathrm{P}=0.297)$ (14). Another North American study involving 697 patients found all patients with a BRAF mutation were former or current smokers $(\mathrm{P}<0.001)(13)$. A large European study included 1,046 patients with NSCLC. This study confirmed a relationship between BRAF mutations and female sex $(\mathrm{P}<0.01)$. This group found all non $\mathrm{BRAF}^{\mathrm{V} 600 \mathrm{E}}$ cases were present in smokers $(\mathrm{P}=0.015)$. Like the other studies the European group reported a shorter disease-free survival, 15.2 vs. 52.1 months $(\mathrm{P}=0.001)$, and overall survival, 29.3 vs. 72.4 months $(\mathrm{P}=0.001)$, in patients with the BRAF ${ }^{\mathrm{V} 600 \mathrm{E}}$ subgroup (12). The non V600E group showed no difference compared to those without a mutation.

The small number of BRAF mutations present in these large studies and variability in findings makes it difficult to be definitive when commenting on a specific clinical 
Table 1 Demographics relative to V600E status and trial reported outcome

\begin{tabular}{|c|c|c|c|c|c|c|}
\hline Trial & $\begin{array}{c}\text { Prevalence } \\
(\%)\end{array}$ & \multicolumn{2}{|c|}{ Sex (\%) } & \multicolumn{2}{|c|}{ Smoking status (\%) } & Outcome (months) V600E \\
\hline Chinese (15) & 1.7 & M: 0.7; F: 0.7 & M: 0; F: 0.2 & Y: 0.4; N: 1.1 & Y: 0; N: 0.2 & PFS: 5.2 vs. $6.4(P=0.561)$ \\
\hline American (14) & 4 & M: 0.9; F: 1.1 & M: 1; F: 1 & Y: $1.5 ; \mathrm{N}: 0.6$ & Y: 1.8; N: 0.2 & PFS: 4.1 vs. $8.9(P=0.297)$ \\
\hline American (13) & 3 & M: 0.3; F: 1 & M: 0.6; F: 0.7 & Y: 1.3; N: 0 & Y: 1.3; N: 0 & NR \\
\hline
\end{tabular}

Figures in bold represent statistically significant findings. M, male; F, female; Y, yes; N, no; PFS, progression free survival; OS, overall survival; NR, not reported.

phenotype. Overall, it would be reasonable to suggest an increased frequency in females. Smoking status and its link with BRAF status varied among studies. Ethnicity and type of BRAF mutation (V600E and non V600E) may be factors that influence this. From the studies outlined, those patients of white Caucasian heritage and positive smoking history were associated with a non V600E mutation. Conversely, a meta-analysis of 16 studies with the V600E subtype was found to be more common in non-smokers (16). What is consistent from these studies is the lack of chemo-sensitivity and worse prognosis in patients with a BRAF ${ }^{\mathrm{V} 6000 \mathrm{E}}$ mutation treated with platinum. Based on these findings it is imperative that alternative strategies are employed for this group.

\section{Treatment options}

\section{Targeted therapy}

The national comprehensive cancer network (NCCN) and European society of medical oncology (ESMO) guidelines now endorse testing for BRAF mutations in NSCLC, in particular $\mathrm{BRAF}^{\mathrm{V} 600 \mathrm{E}}$. These guidelines recommend the use of BRAF/MEK inhibitors in first or subsequent lines of therapy for those that harbour a V600E mutation. The ESMO guidelines do not recommend a specific method of testing a patients BRAF status. The guidelines instead suggest ensuring adequate sensitivity of the test used with appropriate quality control measures in place to ensure its validity. Alternatively, the NCCN guidelines recommend that BRAF, and other molecular testing, should be performed as part of a broader molecular profile $(17,18)$. The data supporting the use of a BRAF inhibitor with or without a MEK inhibitor in BRAF positive NSCLC has been adopted from a small number of positive phase 2 studies.
Single agent Dabrafenib was initially tested in $\mathrm{BRAF}^{\mathrm{V} 600 \mathrm{E}}$ mutated NSCLC. This phase 2 study included 84 patients, 78 pretreated and 6 untreated patients. A 33\% objective response rate (ORR) to Dabrafenib was observed in the pretreated group with a further $24 \%$ categorised as stable disease. The median progression free survival was 5.5 months. 4 of the 6 untreated patients responded to treatment. Grade 3, 4, 5 events occurred in 39\%, 5\% and $1 \%$ respectively, however most cases were considered to be acceptable (19). The combination of Dabfrafenib and Trametinib was evaluated in a second cohort of 57 previously treated patients with $\mathrm{BRAF}^{\mathrm{V} 600 \mathrm{E}}$ mutated NSCLC. The ORR was $63 \%$ with the combination and a further $16 \%$ had stable disease. The median progression free survival was improved to 9.7 months when compared to the single agent Dabrafenib cohort. Adverse events (AE) were common, $98 \%$ of patients experienced an $\mathrm{AE}$ with $49 \%$ experiencing a grade 3 or 4 event. The toxicity profile was considered manageable with modifications to dosing levels and treatment interruptions. Overall, $58 \%$ and $75 \%$ of patients received greater than $80 \%$ of the expected Dabrafenib and Trametinib course respectively (20). Updated survival for the 2 pretreated cohorts demonstrated continued superiority of combination BRAF/MEK inhibition over a single agent. ORR rate for the combination had increased to $67 \%$ as did the median progression free survival, 10.2 months. Median overall survival was reported as 12.7 months in the Dabrafenib only cohort versus 18.2 months in the Dabrafenib and Trametinib cohort (21). Dabrafenib and trametinib was assessed in 36 untreated patients in a separate phase 2 study. ORR in this study was $64 \%$ and median progression free survival of 10.9 months. Adverse events were similar to the pretreated group, all patients experienced an $\mathrm{AE}$ of any grade with $69 \%$ experiencing a grade 3 or 4 event (22). 
Table 2 Patient outcomes

\begin{tabular}{lcc}
\hline Trial & ORR (\%) & PFS (months) \\
\hline Dabrafenib (19) & 33 & 5.5 \\
Dabrafenib and trametinib (20) & 67 & 10.2 \\
Dabrafenib and trametinib-untreated (22) & 64 & 10.9 \\
Vemurafenib (23) & 42 & 7.3 \\
\hline
\end{tabular}

ORR, objective response rate; PFS, progression free survival.

Vemurafenib was tested as a single agent in a basket study of $\mathrm{BRAF}^{\mathrm{V} 600 \mathrm{E}}$ non melanoma cancers. Twenty patients with BRAF $^{\mathrm{V} 600 \mathrm{E}}$ positive NSCLC were included in this study. ORR in this group was $42 \%$ and median progression free survival was 7.3 months (23) (Table 2).

\section{Immunotherapy}

Little is known regarding the effectiveness of immunotherapy in the setting of BRAF positive NSCLC. A retrospective multi-centre chart review was performed to assess response of BRAF positive NSCLC to immune checkpoint inhibitors. Patients were stratified by programmed death ligand 1 (PD-L1) status, tumour mutational burden (TMB) and microsatellite instability (MSI) in 39 patients. Not all patients were tested equally for each of these markers. Twentytwo received an immune check point inhibitor: $57 \%$ of the V600E group and $55 \%$ of the non V600E group. ORR was $25 \%$ and $33 \%$ and median progression free survival 3.7 and 4.1 months respectively. The authors concluded BRAF mutated NSCLC was more likely to have high expression of PD-L1. They also reported that PD-L1 status and BRAF mutation type did not alter outcome. These findings are intriguing however due to the limited number of cases exposed to immune checkpoint inhibitors it is difficult to be conclusive (24). The efficacy of immune checkpoint inhibitor based on PD-L1 expression in patients with oncogenic driver mutations was evaluated in a Japanese population. Five patients were found to have a BRAF mutated NSCLC. No responses were observed in the BRAF cohort (25). A case report described the sequential use of BRAF inhibition followed by Pembrolizumab in a patient with $\mathrm{BRAF}^{\mathrm{V} 600 \mathrm{E}}$ mutated, PD-L1 high $(90 \%)$ NSCLC. In this case the patients achieved an initial 18-month period of disease stability on Dabrafenib and on progression a further durable response to 2 doses of pembrolizumab (stopped due to toxicity). This case highlights the successful application of these drugs in this situation (26).

\section{Conclusions}

BRAF mutated lung cancer is a rare form of NSCLC. Many different types of BRAF mutations have been described with current data focusing on the most common variant, $\mathrm{BRAF}^{\mathrm{V} 600 \mathrm{E}}$. Phase 2 data now exists that confirms activity of BRAF/MEK inhibition as an effective therapy for BRAF ${ }^{\mathrm{V} 600 \mathrm{E}}$ NSCLC in first and subsequent lines of therapy. This is of particular importance based on poor outcomes seen in patients harbouring $\mathrm{BRAF}^{\mathrm{V} 600 \mathrm{E}}$ treated with chemotherapy. No data exists to support the use of BRAF/MEK inhibition for non $\mathrm{BRAF}^{\mathrm{V} 600 \mathrm{E}}$ mutated lung cancer and chemotherapy or immunotherapy remain the favoured options in this case. In contrast to other NSCLC with targets (EGFR, ALK) immune checkpoint inhibitors appear to be active in those with a BRAF mutation irrespective of PD-L1 status or BRAF mutation type. Data in this setting is limited and would need further investigation to clarify this point.

\section{Acknowledgments}

None.

\section{Footnote}

Conflicts of Interest: The authors have no conflicts of interest to declare.

Ethical Statement: The authors are accountable for all aspects of the work in ensuring that questions related to the accuracy or integrity of any part of the work are appropriately investigated and resolved.

\section{References}

1. Rosell R, Carcereny E, Gervais R, et al. Erlotinib versus standard chemotherapy as first-line treatment for European patients with advanced EGFR mutation-positive 
non-small-cell lung cancer (EURTAC): a multicentre, open-label, randomised phase 3 trial. Lancet Oncol 2012;13:239-46.

2. Shaw AT, Kim DW, Nakagawa $\mathrm{K}$, et al. Crizotinib versus chemotherapy in advanced ALK-positive lung cancer. $\mathrm{N}$ Engl J Med 2013;368:2385-94.

3. Shaw AT, Ou SH, Bang YJ, et al. Crizotinib in ROS1rearranged non-small-cell lung cancer. N Engl J Med 2014;371:1963-71.

4. Soria JC, Ohe Y, Vansteenkiste J, et al. Osimertinib in Untreated EGFR-Mutated Advanced Non-Small-Cell Lung Cancer. N Engl J Med 2018;378:113-25.

5. Amaral T, Sinnberg T, Meier F, et al. The mitogenactivated protein kinase pathway in melanoma part I Activation and primary resistance mechanisms to BRAF inhibition. Eur J Cancer 2017;73:85-92.

6. Acosta AM, Kadkol SS. Mitogen-Activated Protein Kinase Signaling Pathway in Cutaneous Melanoma: An Updated Review. Arch Pathol Lab Med 2016;140:1290-6.

7. Okimoto RA, Lin L, Olivas V, et al. Preclinical efficacy of a RAF inhibitor that evades paradoxical MAPK pathway activation in protein kinase BRAF-mutant lung cancer. Proc Natl Acad Sci U S A 2016;113:13456-61.

8. Amaral T, Sinnberg T, Meier F, et al. MAPK pathway in melanoma part II-secondary and adaptive resistance mechanisms to BRAF inhibition. Eur J Cancer 2017;73:93-101.

9. Rudin CM, Hong K, Streit M. Molecular characterization of acquired resistance to the BRAF inhibitor dabrafenib in a patient with BRAF-mutant non-small-cell lung cancer. J Thorac Oncol 2013;8:e41-2.

10. Spagnolo F, Ghiorzo P, Queirolo P. Overcoming resistance to BRAF inhibition in BRAF-mutated metastatic melanoma. Oncotarget 2014;5:10206-21.

11. Jain P, Silva A, Han HJ, et al. Overcoming resistance to single-agent therapy for oncogenic BRAF gene fusions via combinatorial targeting of MAPK and $\mathrm{PI} 3 \mathrm{~K} / \mathrm{m}$ TOR signaling pathways. Oncotarget 2017;8:84697-713.

12. Marchetti A, Felicioni L, Malatesta S, et al. Clinical features and outcome of patients with non-small-cell lung cancer harboring BRAF mutations. J Clin Oncol 2011;29:3574-9.

13. Paik PK, Arcila ME, Fara M, et al. Clinical characteristics of patients with lung adenocarcinomas harboring BRAF mutations. J Clin Oncol 2011;29:2046-51.

14. Cardarella S, Ogino A, Nishino M, et al. Clinical, pathologic, and biologic features associated with BRAF mutations in non-small cell lung cancer. Clin Cancer Res
2013;19:4532-40.

15. Ding X, Zhang Z, Jiang T, et al. Clinicopathologic characteristics and outcomes of Chinese patients with nonsmall-cell lung cancer and BRAF mutation. Cancer Med 2017;6:555-62.

16. Cui G, Liu D, Li W, et al. A meta-analysis of the association between BRAF mutation and nonsmall cell lung cancer. Medicine (Baltimore) 2017;96:e6552.

17. NCCN.org. NCCN Clinical Practice Guidelines in Oncology (NCCN Guidelines®) Non-Small Cell Lung Cancer. NCCN Evidence Blocks. 2018; Version 6.2018 August 17, 2018.

18. Planchard D, Popat S, Kerr K, et al. Metastatic non-small cell lung cancer: ESMO Clinical Practice Guidelines for diagnosis, treatment and follow-up. Ann Oncol 2018;29:iv192-237.

19. Planchard D, Kim TM, Mazieres J, et al. Dabrafenib in patients with BRAF(V600E)-positive advanced non-smallcell lung cancer: a single-arm, multicentre, open-label, phase 2 trial. Lancet Oncol 2016;17:642-50.

20. Planchard D, Besse B, Groen HJM, et al. Dabrafenib plus trametinib in patients with previously treated BRAF(V600E)-mutant metastatic non-small cell lung cancer: an open-label, multicentre phase 2 trial. Lancet Oncol 2016;17:984-93.

21. Planchard D, Besse B, Kim TM, et al. Updated survival of patients (pts) with previously treated BRAF V600Emutant advanced non-small cell lung cancer (NSCLC) who received dabrafenib (D) or D + trametinib (T) in the phase II BRF113928 study. J Clin Oncol 2017;35:9075.

22. Planchard D, Smit EF, Groen HJM, et al. Dabrafenib plus trametinib in patients with previously untreated BRAF(V600E)-mutant metastatic non-small-cell lung cancer: an open-label, phase 2 trial. Lancet Oncol 2017;18:1307-16.

23. Hyman DM, Puzanov I, Subbiah V, et al. Vemurafenib in Multiple Nonmelanoma Cancers with BRAF V600 Mutations. N Engl J Med 2015;373:726-36.

24. Dudnik E, Peled N, Nechushtan H, et al. BRAF Mutant Lung Cancer: Programmed Death Ligand 1 Expression, Tumor Mutational Burden, Microsatellite Instability Status, and Response to Immune Check-Point Inhibitors. J Thorac Oncol 2018;13:1128-37.

25. Yoh K, Matsumoto S, Kunimasa K, et al. The efficacy of immune checkpoint inhibitors and PD-L1 status in patients with advanced non-small cell lung cancer harboring oncogenic driver alterations: Immuno-oncology biomarker study in LC-SCRUM-Japan. J Clin Oncol 
2019;37:abstr 9046.

26. Li SD, Martial A, Schrock AB, et al. Extraordinary clinical benefit to sequential treatment with targeted therapy and

Cite this article as: O'Leary CG, Andelkovic V, Ladwa R, Pavlakis N, Zhou C, Hirsch F, Richard D, O’Byrne K. Targeting BRAF mutations in non-small cell lung cancer. Transl Lung Cancer Res 2019;8(6):1119-1124. doi: 10.21037/ tlcr.2019.10.22 immunotherapy of a BRAF V600E and PD-L1 positive metastatic lung adenocarcinoma. Exp Hematol Oncol 2017;6:29. 